\title{
Adaptive Variable Step-Size Algorithm for Acoustic Noise Cancellation by using Multiple Sub-Filters Approach
}

\author{
Amira A. Mohamed \\ Faculty of Engineering, Ain \\ Shams University \\ Cairo, Egypt
}

\author{
Waleed M. El-Nahal \\ Faculty of Engineering, Modern \\ Science and Arts University \\ October City, Egypt
}

\author{
Adel E. El-Hannawy \\ Faculty of Engineering, Ain \\ Shams University \\ Cairo, Egypt
}

\begin{abstract}
In this paper, a novel algorithm for cancelling noise from the speech signal in real time environment is proposed. Least Mean Square (LMS) adaptive noise cancellers are often used to recover signal corrupted by additive noise due to its simplicity in implementation. But it has limitation when the desired signal is strong, that the excess mean-square errors increase linearly with the desired signal power. This results in downgraded performance when the desired signal exhibits large power fluctuations. In the proposed algorithm we use the benefits of both variable step size (VSS) LMS algorithm and Normalized Differential LMS (NDLMS) algorithm to deal with this situation. One more addition of this algorithm is that it uses the concept of decomposing the long adaptive filter into low order multiple sub-filters to relieve the effect of slow convergence of that long adaptive filter. Finally, The proposed (P-VSSNDLMS) algorithm yields faster convergence with minimum mean square error in simulations which carried out using real speech signal with different noise power levels.
\end{abstract}

\section{Keywords}

Adaptive Noise canceller (ANC), mean square error (MSE), VSSNDLMS, multiple sub-filters.

\section{INTRODUCTION}

The goal of any filter is to extract useful information from noisy data. A normal fixed filter is designed in advance with knowledge of the statistics of both signal and the unwanted noise but if the statistics of the noise are not known priori, or change over time, the coefficients of the filter cannot be specified in advance. In these situations, adaptive algorithms are needed in order to continuously update the filter coefficient [7].

Adaptive filtering finds application in noise cancellation in speech called as Adaptive Noise cancellation (ANC) which involves in time-varying signals and systems. ANC is an effective method for recovering a signal corrupted by additive noise [1] and it is an important core area of the digital signal processing [2]. The typical noise cancellation scheme is shown in Fig 1.

Fig 1 shows the basic problem and the adaptive noise cancelling solution to it. A signal $\mathrm{s}(\mathrm{n})$ is transmitted over a channel to a sensor that also receives a noise $\mathrm{n}_{0}$ uncorrelated with the signal. The primary input to the canceller is combination of both signal and noise $\mathrm{s}+\mathrm{n}_{0}$. A second sensor receives a noise $n_{1}$ uncorrelated with the signal but correlated with the noise $\mathrm{n}_{0}$. This sensor provides the reference input to the canceller. This noise $n_{1}$ is filtered to produce an output $y(n)$ that is as close a replica of $n_{0}$ [9]. This output of the adaptive filter is subtracted from the primary input to produce the adaptive filter error $e(n)=d(n)-y(n)$.

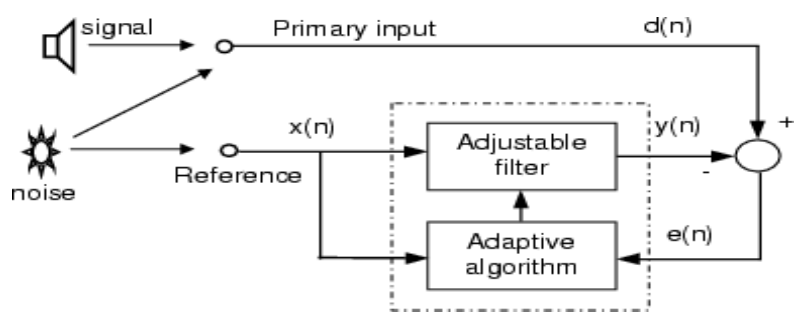

Fig 1: Adaptive noise canceller

Probably, one of the well-known algorithms in the field of adaptive filtering is the Least Mean Square (LMS) algorithm. Simplicity and easy implementation are the main reasons for the popularity of LMS algorithm. Some successful applications of the LMS filters are: system identification, channel equalization, echo cancellation and it has been widely used in noise cancellation [3].

There are numerous adaptive algorithms have been developed for noise cancellation. J Zhang and H.M Tai are proposed a Normalized Differential LMS algorithm [5] to improve the steady state performance for cancelling noise in speech processing. J.E. Greenberg has proposed a modified LMS to cancel noise in speech signal [1]. A new variable length LMS algorithm has developed by R.Bilcu [3]. Gorriz et.al has introduced a novel LMS algorithm for filtering speech sounds in the adaptive noise cancellation [4]. M.A. Raja and A.Shanmugam are proposed a new approach called Variable step size Normalized differential LMS to remove noise from the speech signal in real time environment [2].

\section{ADAPTIVE ALGORITHMS}

Several modified LMS algorithms have been proposed in the past years in order to simultaneously improve the tracking ability and speed of convergence of corrupted signal.

They provide an extensive performance evaluation Compared to standard LMS algorithms, including the NDLMS, VSSNLMS and other recently reported LMS algorithms such as the VSSNDLMS.

In this paper, we analyzed these algorithms and a new approach named Parallel Variable Step Size Normalized Difference LMS (P- VSSNDLMS) is proposed using the concept decomposing the long adaptive filter into low order multiple sub- filters in which the error signals are independent on each other. The independency of the error signals exhibits the parallelism technique. This achieves our goal in increasing speed of the convergence rate. Simulation results show that the proposed decomposed Variable step size Normalized Differential LMS adaptive algorithm significantly improves the convergence rate and minimizes mean square error with 
respect to that of the original long VSSNDLMS adaptive filter.

\subsection{LMS Algorithm}

One of the simplest algorithms used in the adaptive filters is LMS algorithm because of its simplicity, ease of implementation, and low computational complexity [1]. Using the LMS algorithm, the weight update equation and error equation for the adaptive noise canceller are given by

$\mathbf{W}(\mathrm{n}+1)=\mathbf{W}(\mathrm{n})+\mu \mathrm{e}(\mathrm{n}) \mathbf{x}(\mathrm{n})$
$\mathrm{e}(\mathrm{n})=\mathrm{d}(\mathrm{n})-\mathbf{W}^{\mathrm{T}}(\mathrm{n}) \mathbf{x}(\mathrm{n})$

Where $\mathrm{n}$ is the time index, boldface characters represent vectors, $\mathbf{W}(\mathrm{n})$ is the $\mathrm{L} \times 1$ vector of adaptive filter weights, $\mathrm{L}$ is the length of the adaptive filter, $(.)^{\mathrm{T}}$ is Transpose of a vector, $\mu$ is a step-size parameter controlling the convergence and the steady state behavior of the LMS. An approximate condition for the convergence is:

$$
0<\mu<\frac{2}{\mathrm{LS}_{\max }}
$$

where $S_{\max }$ is the maximum value of the power spectral density of the received vector [8].

One major disadvantage of the adaptive noise canceller using the LMS algorithm is that the steady-state excess mean square error (MSE), which increases linearly with the desired signal power. This leads to degrading performance when the desired signal exhibits large power fluctuations and is a serious problem in many speech processing applications [1].

\subsection{NDLMS Algorithm}

A normalized difference LMS (NDLMS) algorithm is proposed to deal with the situation when the desired signal is strong, e.g., speech signals [5], which is a major drawback of conventional LMS algorithms.

This algorithm adjusts the adaptive filter weights $\mathrm{W}$ (n) using the difference reference input $\nabla \times(n)$ and the difference output $\nabla$ e(n) instead of $x(n)$ and e(n), respectively, as shown in Fig 2. [5]

The weight update equation becomes [2]

$$
\begin{aligned}
& \mathrm{W}(\mathrm{n}+1)=\mathrm{W}(\mathrm{n})+\mu \nabla \mathrm{x}(\mathrm{n}) \nabla \mathrm{d}(\mathrm{n}) \\
& \mathrm{e}(\mathrm{n})=\mathrm{d}(\mathrm{n})-\mathrm{W}^{\mathrm{T}}(\mathrm{n}) \mathrm{x}(\mathrm{n}) \mathrm{d}(\mathrm{n})
\end{aligned}
$$

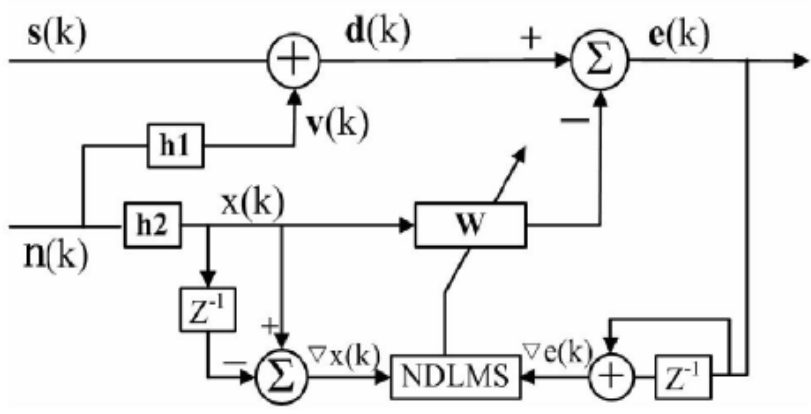

Fig 2: Adaptive noise canceller with normalized difference LMS algorithm

NDLMS algorithm produces very small error compared with the conventional LMS and NLMS methods. This algorithm also improves steady state performance of adaptive noise cancellers for speech processing [5].

\subsection{VSSNLMS Algorithm}

The NDLMS algorithm exhibits a good balance between computational cost and performance. However, a very serious problem associated with both the LMS and NDLMS algorithms is the choice of the step-size $(\mu)$ parameters. A small step size (small compared to the reciprocal of the input signal strength) will ensure small mis-adjustments in the steady state, but the algorithms will converge slowly and may not track the non-stationary behavior of the operating environment very well. On the other hand a large step size will in general provide faster convergence and better tracking capabilities at the cost of higher misadjustment. Any selection of the step-size must therefore be a trade-off between the steady-state misadjustment and the speed of adaptation [10].

VSSNLMS algorithm overcomes the problem of convergence speed and estimation accuracy in real time environment. The signal to noise (SNR) ratio is defined as the ratio of the average power of the original signal to that of the noise signal. The main aim of proposing this algorithm is that with the help of SNR, the step size adjustment can be controlled. It is efficient to have lesser value of SNR because such a value gives the maximized step size that provides faster tracking. At the same time, the larger value of SNR results in minimized step size producing smaller mis-adjustment [2].

To estimate the SNR, the average power of the speech signal $\mathrm{P}_{\mathrm{S}}(\mathrm{n})$ and the noise signal $\mathrm{P}_{\mathrm{N}}(\mathrm{n})$ respectively are considered as,

$$
\begin{aligned}
& \mathrm{P}_{\mathrm{S}}(\mathrm{n})=\sum_{j=0}^{M-1}[\mathrm{~s}(\mathrm{n}-1)-\mathrm{u}(\mathrm{n}-\mathrm{j})]^{2} \\
& \mathrm{P}_{\mathrm{N}}(\mathrm{n})=\sum_{j=0}^{M-1} \mathrm{y}^{2}(\mathrm{n}-\mathrm{j})
\end{aligned}
$$

Where, $P_{S}(n)$ and $P_{N}(n)$ can be estimated by the number of samples $\mathrm{M}$ from equation (6) and (7).SNR (n) is given as

$\operatorname{SNR}(n)=10 \log _{10} \frac{P_{S}(n)}{P_{N}(n)} d B$

The updating of the normalized coefficients is given by

$\mathrm{w}(\mathrm{n}+1)=\mathrm{w}(\mathrm{n})+\frac{\mu}{\mathrm{X}^{\mathrm{T}}(\mathrm{n}) \mathrm{X}(\mathrm{n})} \mathrm{e}(\mathrm{n}) \mathrm{X}(\mathrm{n})$

When the value of SNR is small, step size will increase and thus leads to fast convergence and when the SNR is high step size $\mu$ will be small. So step size and SNR values are interrelated. The following equation determines the step size $\mu$ [2].

$$
\mu=\left\{\begin{array}{cc}
\mu_{\min } & \text { if } \mathrm{SNR}(\mathrm{n})>\mathrm{SNR}_{\max } \\
\mu_{\max } & \text { if } \operatorname{SNR}(\mathrm{n})>\mathrm{SNR}_{\min } \\
\mathrm{aSNR}(\mathrm{n})+\mathrm{b} & \text { if } \mathrm{SNR}_{\text {min }} \leq \mathrm{SNR}(\mathrm{n}) \leq \mathrm{SNR}_{\max }
\end{array}\right.
$$

Thus, this algorithm uses a variable step size instead of fixed step size used by NDLMS algorithm. As a result, the convergence rate of this algorithm is significantly faster than that of NDLMS algorithm [10].

\subsection{VSSNDLMS Algorithm}

VSSNDLMS is purposed by M.A.RAJA and A.SHANMUGAM [2] to remove the noise in signal recorded in various environments like in street or inside a car. The variable step size LMS algorithm converges fastly and NDLMS algorithm has minimized mean square error. This algorithm combines VSS and NDLMS; In this case, the 
VSSNDLMS algorithm converges fastly with minimum mean square error.

The expression for updating the Coefficient is given by

$\mathbf{w}(\mathrm{n}+1)=\mathbf{w}(\mathrm{n})+\frac{\mu_{\mathrm{var}}}{\varepsilon+\|\nabla \mathbf{X}(\mathrm{k})\|^{2}} \nabla \mathbf{x}(\mathrm{n}) * \nabla \mathrm{e}(\mathrm{n}) \ldots$

where $\|\nabla \mathbf{X}(\mathrm{k})\|^{2}$ denote the Euclidean norm of the input vector $\mathrm{X}(\mathrm{n})$. And

$\nabla \mathrm{e}(\mathrm{k})=\mathrm{e}(\mathrm{k})-\mathrm{e}(\mathrm{k}-1)$

$\nabla \mathrm{x}(\mathrm{k})=\mathrm{x}(\mathrm{k})-\mathrm{x}(\mathrm{k}-1)$

And the $\mu_{\mathrm{var}}$ is the variable step size which is given by

$\mu_{\mathrm{var}}= \begin{cases}\mu_{\max } & \text { if } \mu_{\mathrm{i}}>\mu_{\max } \\ \mu_{\min } & \text { if } \mu_{\mathrm{i}}<\mu_{\min }\end{cases}$

Otherwise $\mu_{\mathrm{var}}=\mu_{\mathrm{i}}$

$\mu_{\mathrm{i}}=\alpha \mu+\gamma \varepsilon$, and

$\alpha=0.97 ; \gamma=4.8 * 10^{-4}$

Where, Spectral density of the tap inputs $x(n)$ and the filter length $\mathrm{M}$ is moderate to large.

\section{PROPOSED ALGORITHM}

In our work we consider a new approach called Parallel Variable step size Normalized Differential LMS (PVSSNDLMS) algorithm. This proposed algorithm uses a way to alleviate the effect of slow convergence of long adaptive filter problem and this is achieved by using Multiple Subfilters (MSF) parallel structure. This algorithm applies the concept of decomposing the long adaptive filter into low order multiple sub-filters to the VSSNDLMS algorithm (see fig. 3) [12]. Simulation results show that using this algorithm increases convergence speed, minimize excess mean square error, reduce misadjustment and increase estimation accuracy.

Here the decomposition is to partition the long single adaptive filter into smaller multiple sub-filters. Each sub filter is updated by an individual adaptive algorithm. Adaptive algorithms are constructed depending upon how the error signal is generated. The error signal can be obtained at each stage of the sub filter for its updating [11].

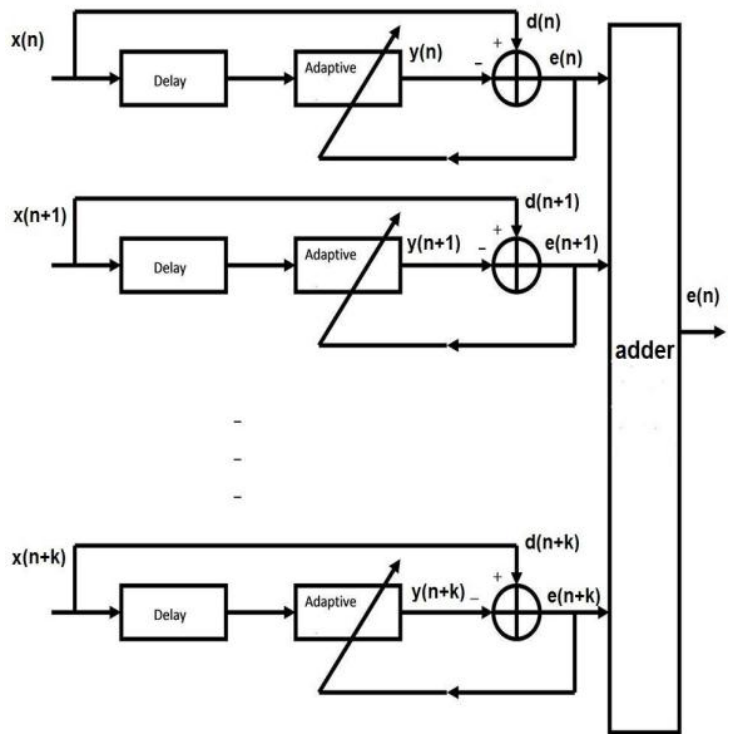

Fig 3: Parallel VSSNDLMS implementation
The equations that describes the proposed algorithm is as follows

$d(n)=\sum_{j=0}^{M} H_{j}^{T} X_{j}(n)+\varepsilon(n)$

where $H_{j}=\left[h_{j, 1}, h_{j, 2}, \ldots, h_{j, L j}\right]^{T}$ is the Wiener solution of the $\mathrm{j}^{\text {th }}$ sub-filter,

$\mathrm{X}_{\mathrm{j}}=\left[\mathrm{x}\left(\mathrm{n}-\left(\mathrm{K}_{1}+\mathrm{K}_{2}+\ldots+\mathrm{K}_{\mathrm{j}}\right)\right), \mathrm{x}\left(-\left(\mathrm{K}_{1}+\mathrm{K}_{2}+\ldots+\right.\right.\right.$

$\left.\left.\left.\mathrm{K}_{\mathrm{j}}+1\right)\right), \ldots, \mathrm{x}\left(-\left(\mathrm{K}_{1}+\mathrm{K}_{2}+\ldots+\mathrm{K}_{\mathrm{j}}+\mathrm{L}_{\mathrm{j}}-1\right)\right)\right]^{\mathrm{T}}$ input

sequence.

$\mathrm{M}$ is the number of iterations taken into account and $\mathrm{Lj}$ is the length of the filter.

The output of the adaptive sub-filter is given by

$y(n)=\sum_{j=0}^{M} W_{j}^{T}(n) X_{j}(n)$

Where $W_{j}(n)=\left[w_{j, 1}(n), w_{j, 2}(n), \ldots ., w_{j, L j}(n)\right]^{T}$

The error signals are defined as

$e_{i}(n)=d(n)-W_{i}^{T}(n) X_{i}(n)$

where $\mathrm{i}=1,2, \ldots ., \mathrm{M}$

The expression for updating the coefficient of each sub-filter is given by

$$
\begin{aligned}
& \mathrm{W}_{\mathrm{i}}(\mathrm{n}+1)=\mathrm{W}_{\mathrm{i}}(\mathrm{n})+\frac{\mu_{\mathrm{var}}}{\varepsilon+\left\|\nabla \mathrm{X}_{\mathrm{i}}(\mathrm{k})\right\|^{2}} \nabla \mathrm{X}_{\mathrm{i}}(\mathrm{n}) \nabla \mathrm{e}_{\mathrm{i}}(\mathrm{n}) \ldots \\
& \mathrm{i}=1,2, \ldots ., \mathrm{M}
\end{aligned}
$$

It is known that the adaptive filters reduces the mean square error (MSE) and it is given as,

$$
\mathrm{MSE}=\mathrm{E}\left\{|\mathrm{d}(\mathrm{n})-\hat{\mathrm{d}}(\mathrm{n})|^{2}\right\}
$$

Therefore, the graph of MSE quantity is essential to evaluate the performance of the adaptive filter [2].

This new proposed algorithm shows through computer simulation good performance compared with other variable step size LMS algorithm.

\section{EXPERIMENTAL RESULTS}

This section describes computer simulations using Matlab7 version to examine the performance enhancement of the proposed algorithm compared with the original long variable step size normalized difference LMS , without using decomposing technique, (VSSNDLMS) algorithm.

Number of tap weights (i.e. $\mathrm{N}$ ) is equal to 40 ; real speech signal $d(n)$ is the desired signal, shown in Fig. 4.a, which it is sampled at $8 \mathrm{KHz}$. Then this signal corrupted by noise and used as input to the adaptive filter $\mathrm{x}$ (n) (Fig. 4.b)

The step size for the used algorithms is equal to 0.001, in order to ensure stability (or convergence) of the algorithm; the step size parameter is bounded by the following equation:

$0<\mu<\frac{2}{\text { tap weight power }}$ 

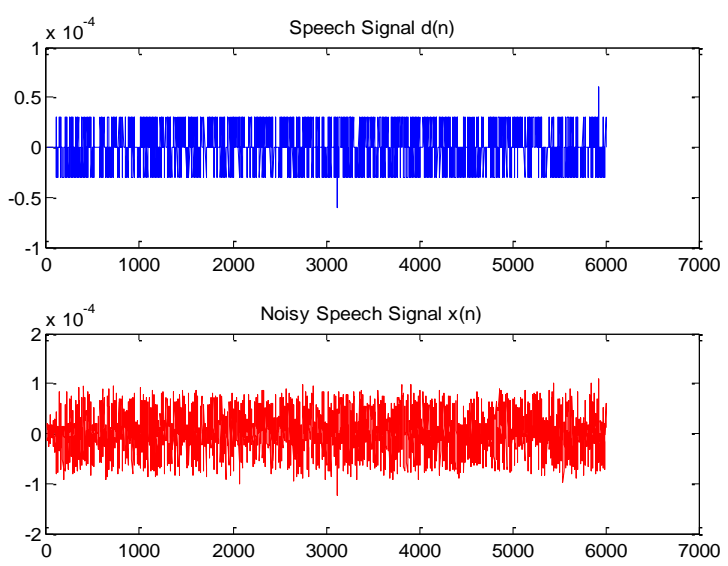

Fig 4: (a) Speech signal d (n); (b) Noisy speech signal $x$ (n)

Fig 5 shows the mean square error (MSE) using different algorithms. It is clearly that the proposed algorithm has the minimum MSE compared with other algorithm.

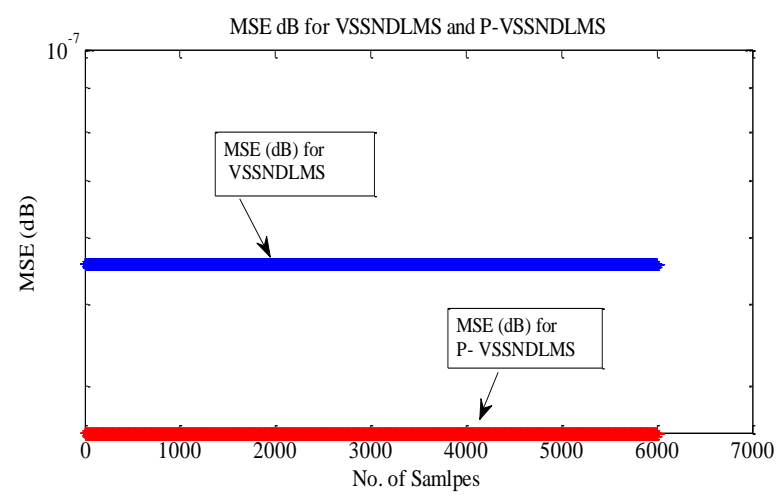

Fig 5: Mean square error (MSE) for VSSNDLMS and PVSSNDLMS

Moreover, Fig 6 shows what happen to MSE while increasing signal power, signal to noise ratio, (SNR). As shown, the proposed algorithm provides an extensive performance in decreasing the mean square error. Therefore the error of the proposed algorithm is the best in decreasing MSE.

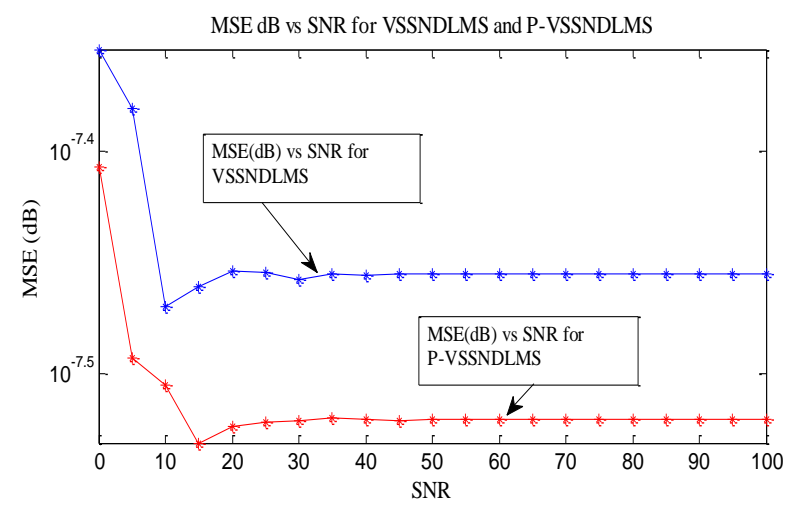

Fig 6: MSE verses SNR for VSSNDLMS and PVSSNDLMS

From the simulation results, it is evident that, the decomposed variable step size LMS (P-VSSNDLMS) adaptive algorithm gives much smaller error and provides good performance than the original long VSSNDLMS algorithm.

\section{CONCLUSION}

This letter introduced a novel P-VSSNDLMS algorithm based on using Multiple Sub-filters (MSF) parallel structure (i.e. decomposing technique) to remove noise from speech signal. This current work has been compared with another algorithm, VSSNDLMS in order to examine the performance enhancement of the proposed algorithm. Speech signals recorded are taken and processed using both algorithms while increasing signal power, signal to noise ratio, (SNR), the simulations show that the behavior of the new algorithm provides an improved performance in decreasing the mean square error and fast convergence compared to referenced algorithm. Hence, the proposed P-VSSNDLMS algorithm is a promising method for noise cancellation.

\section{REFERENCES}

[1] J. E. Greenberg, "Modified LMS algorithms for speech processing with an adaptive noise canceller", IEEE Trans. Speech audio process. vol 6, no4, pp 338-358 July 1998.

[2] M.A.RAJA, Dr.A.SHANMUGAM, "Performance analysis of adaptive algorithms For noise cancellation in speech processing", Journal of Theoretical and Applied Information Technology, 2013, Vol. 48 No.2

[3] R.Bilcu, P.Kuosmanen, K.Egiazarian, A new variable length LMS algorithm: theoretical analysis and implementation, $9^{\text {th }}$ International conference on Electronics, Circuits and Systems, Vol.3, 2002, pp.10311034

[4] Gorriz, J.M. Ramirez, J. Cruces-Alvarez, S. Puntonet, C.G. Lang, E.W. Erdogmus, "A novel LMS algorithm applied to adaptive noise cancellation", IEEE signal Processing Letters Vol 16, pp 34-37.

[5] Jiashu Zhang and Heng-Ming Tai, "Adaptive Noise Cancellation Algorithm for Speech Processing", the $33^{\text {rd }}$ Annual Conference of User the IEEE Industrial Electronics Society. (IECON) Taipei, Taiwan.

[6] R.N. Sharma, A.K. Chaturvedi and G. Sharma, "Acoustic echo cancellation using multiple sub-filters", IIT Kanpur.

[7] Joseph Petrone, “Adaptive Filter Architectures for FBGA Implementation “, June 2004.

[8] Ran Meng," Sparsity-aware Adaptive Filtering Algorithms and Applications to System Identification", December 2011.

[9] Jashvir Chhikara, Jabir Singh, "Noise Cancellation using Adaptive Algorithms", International Journal of Modern Engineering Research (IJMER), Vol.2, Issue.3, MayJune 2012 pp-792-795

[10] Oyerinde and S.H. Mneney, “Variable Step Size Algorithms for Network Echo Cancellation" , UbiCC Journal, Volume 4, Number 3, August 2009

[11] Amin M. Nassar, Ashraf M. Ali, "Multiple Sub-Filters Approach to Acoustic Echo Cancellation “ , 25th National Radio Science Conference (NRSC), March 2008

[12] M. Jaber , "The Ultra High Speed LMS Algorithm Implemented on Parallel Architecture Suitable for Multidimensional Adaptive Filtering" US patent No. $7,533,140,2009$ 